\title{
Vorhersage langfristiger Behinderungen
}

Fragestellung: Wie ist die langfristige Zunahme der Behinderung in einer behandelten Kohorte von Patienten mit Multipler Sklerose (MS) und haben klinische und magnetresonanztomografische Parameter eine prognostische Aussagekraft?

Hintergrund: Die heute verfügbaren Immuntherapien haben das Risiko einer Behinderung signifikant reduziert. Die Beobachtungsintervalle beschränken sich aber auf die wenigen Jahre der jeweiligen Zulassungsstudien und es ist unklar, ob die bisher verwendeten klinischen und magnetresonanztomografischen Verlaufsparameter auch langfristige Verlaufsprädiktoren sind.

Patienten und Methodik: Es handelte sich um eine prospektive monozentrische Studie mit 517 MS-Patienten. Über einen Zeitraum bis zu zehn Jahren wurden die EDSS (Expanded Disability Status Scale), der T25W (Timed 25-Foot Walk), der 9-HPT (9-Hole-PEG-Test) und der PASAT-3 (Paced Auditory Serial Addition Test) sowie Magnetresonanztomografie(MRT)-Aufnahmen inklusive Volumetrie jährlich durchgeführt. Zusätzlich wurden zu Beginn, im ersten und im zweiten Jahr der Studie die VitaminD-Spiegel bestimmt. Die Kohorte wurde stratifiziert nach der Verlaufsform (klinisch isoliertes Syndrom [ $\mathrm{n}=82$ )] plus schubförmige MS [ $n=366$ ] als RMS-Patienten sowie primär $(n=21)$ plus sekundär chronisch progrediente MS [ $\mathrm{n}=48$ ] als

Cree BA, Gourraud PA, Oksenberg JR et al. Long-term evolution of multiple sclerosis disability in the treatment era. Ann Neurol 2016; 80: 499-510
PMS-Patienten) und der Behandlungsform (Plattformtherapiegruppe: InterferonPräparate, Glatirameracetat, Azathioprin, Mycophenolat
Mofetil, Kortisonstoßtherapie; hochpotente Therapiegruppe: Natalizumab, Rituximab, Mitoxantron, Cyclophosphamid). Statistisch wurde die Zeit bis zu einem EDSS von 6 oder bis zur Konversion in eine sekundär chronisch progrediente Verlaufsform als auch die Assoziation zwischen langfristig erlittenen Behinderungen und den erhobenen Parametern ermittelt.

Ergebnisse: Das zweite und das zehnte Studienjahr wurden von 489 beziehungsweise 471 Patienten beendet. Bei 225 RMS-Patienten (55,3\%) traten EDSS-Verschlechterungen auf. Mehr als 75\% der PMS-Patienten, bei einer Ausgangs-EDSS von $<3$ sogar $100 \%$, erlitten EDSS-Verschlechterungen. Schätzungen zufolge werden $4,7 \%$ und $16,2 \%$ der RMS-Patienten eine EDSS von $\geq 6$ nach zehn beziehungsweise 20 Jahren erreichen. Bei 6,4\% und 24,2\% dieser Patienten wird nach zehn beziehungsweise 20 Jahren die Erkrankung einen chronisch progredienten Verlauf nehmen. Das Vorliegen von „No Evidence of Disease Activity“ (NEDA) im zweiten Studienjahr hatte keinen Einfluss auf die Zehnjahres-EDSS. Frühe MRT-Aktivität bis zum zweiten Studienjahr, die Vitamin-DWerte in den ersten zwei Studienjahren, eine Therapieeskalation oder die Zugehörigkeit zu einer der Behandlungsgruppen waren nicht signifikant mit den im zehnten Studienjahr ermittelten $\mathrm{Pa}-$ rametern (EDSS, T25W, 9HPT und PASAT-3) assoziiert.

Schlussfolgerungen: Die Autoren folgern, dass frühe klinische und radiologische Veränderungen und das Vorliegen von NEDA keine prädiktive Bedeutung für den langfristigen Krankheitsverlauf besitzen. Ihre Kohorte weist gegenüber früheren MS-Verlaufsstudien eine geringere Rate der klinischen Verschlechterung sowie Konversion zur chronisch progredienten Verlaufsform auf.

\section{- Kommentar von Refik Pul und Christoph Kleinschnitz, Essen}

\section{Weitere Langzeitkohortenstudien bleiben abzuwarten}

Die vorliegenden Ergebnisse ähneln denen aus einer neueren sieben Jahre umfassenden Verlaufsstudie von Beta-Interferonbehandelten MS-Patienten [1]. Wahrscheinlich wurde in Anlehnung an diese Arbeit der Begriff „Behandlungsära“ im Titel aufgenommen, obgleich 155 RMS- $(38,1 \%)$ und 28 PMS-Patienten $(43,8 \%)$ unbehandelt waren. Der Datengenerierung liegt eine umfangreiche, komplexe Statistik zugrunde. Unklar bleibt allerdings, bei wie vielen Patienten überhaupt eine Therapieeskalation durchgeführt wurde. Provokant ist die Fragestellung, ob klinische (EDSS, T25W, 9-HPT und PASAT-3) und/ oder radiologische Veränderungen (neue T2-Läsionen und ihre Größenzunahme) als auch NEDA als Kompositfaktor, die innerhalb eines kurzen Zeitraumes von zwei Jahren ermittelt wurden, den langfristigen Verlauf vorhersagen können. Die Autoren kommen zu einem eher ernüchternden Ergebnis und stellen damit nicht nur herkömmliche Konzepte von Zulassungsstudien, sondern bezogen auf das MRT auch gegenwärtige Therapieentscheidungen infrage. Das Ergebnis über
NEDA sollte bei einer Anzahl von 73 Patienten aber kritisch betrachtet werden. Bevor nun alle etablierten klinischen und radiologischen Parameter infrage gestellt werden, sollten weitere Langzeitkohortenstudien abgewartet werden.
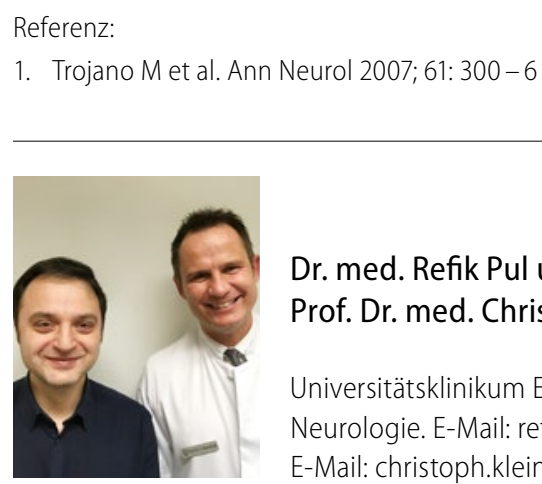
Dr. med. Refik Pul und Prof. Dr. med. Christoph Kleinschnitz

Universitätsklinikum Essen, Klinik für Neurologie. E-Mail: refik.pul@uk-essen.de E-Mail: christoph.kleinschnitz@uk-essen.de 